\title{
Article
}

\section{Effects of different heel heights on lower extremity joint loading in experienced and in-experienced users: $A$ musculoskeletal simulation analysis}

Sinclair, Jonathan Kenneth, Brooks, Darrell and Butters, Bobbie Available at http://clok.uclan.ac.uk/25781/

Sinclair, Jonathan Kenneth ORCID: 0000-0002-2231-3732, Brooks, Darrell ORCID: 0000-0002-4094-5266 and Butters, Bobbie (2019) Effects of different heel heights on lower extremity joint loading in experienced and inexperienced users: A musculoskeletal simulation analysis. Sport Sciences for Health . ISSN 1824-7490

It is advisable to refer to the publisher's version if you intend to cite from the work. http://dx.doi.org/10.1007/s11332-019-00534-4

For more information about UCLan's research in this area go to http://www.uclan.ac.uk/researchgroups/ and search for <name of research Group>.

For information about Research generally at UCLan please go to http://www.uclan.ac.uk/research/

All outputs in CLoK are protected by Intellectual Property Rights law, including Copyright law. Copyright, IPR and Moral Rights for the works on this site are retained by the individual authors and/or other copyright owners. Terms and conditions for use of this material are defined in the policies page. 
1 Effects of different heel heights on lower extremity joint loading in experienced and in-

\section{Correspondence Address:}

9 Dr. Jonathan Sinclair

10 Centre for Applied Sport Exercise and Nutritional Sciences

11 Faculty of Health and Wellbeing

12 University of Central Lancashire

13 Preston

14 Lancashire

PR1 2HE.

e-mail: jksinclair@uclan.ac.uk

Keywords: Biomechanics; high-heels; osteoarthritis; musculoskeletal Central Lancashire, Lancashire, UK. 


\section{Abstract}

23 Purpose: This study examined the effects of different high-heeled footwear heights on lower extremity compressive joint loading and triceps-surae muscle tendon kinematics during walking, using a musculoskeletal simulation based approach, in both experienced and inexperienced high heel users.

Methods: The current investigation examined 12 experienced and 12 inexperienced highheel wearers, walking in four different footwear (high heel, medium heel, low heel and trainer). Walking kinematics were collected using an eight-camera motion capture system, and kinetics via an embedded force plate. Lower extremity joint loading and triceps-surae muscle kinematics were explored using a musculoskeletal simulation approach.

Results: Irrespective of experience, when wearing high-heels of increasing height, compressive loading parameters at the medial tibiofemoral compartment and patellofemoral joint were significantly greater and exceeded the minimum clinically important difference (MCID). Furthermore, irrespective of wearers' experience, the triceps-surae muscle tendon units were placed in a shortened position when wearing high-heels of increasing height, with the differences exceeding the MCID.

Conclusions: It can be concluded that heeled-footwear increase the mechanical factors linked to the aetiology of degenerative joint osteoarthritis, and chronic shortening of the tricepssurae muscle tendon units. Therefore, the current investigation provides evidence that irrespective of experience, heeled-footwear of increasing height may negatively influence female's lower extremity musculoskeletal health. 
Walking is a fundamental aspect of everyday living, and the principal locomotion modality in humans. High-heeled shoes have been a prevalent footwear choice for over 400 years and are used daily in 39-69\% of females (1). Heeled designs remain one of the central features of women's footwear, and social and fashion practices promote the continued use of high-heels (2). Although millions of women wear heeled footwear, concerns regarding the chronic impact of high-heels on women's musculoskeletal health have been articulated for over 50 years (1).

High-heeled footwear feature a slender base of support, and force the ankle in to a plantarflexed state, mediating kinematic and kinetic changes in lower extremity biomechanics during walking (3). A substantial literature base currently exists concerning the biomechanics of walking in heeled footwear. Stefanyshyn et al., (4) examined heel heights of 1.4, 3.7, 5.4 and $8.5 \mathrm{~cm}$ and showed firstly that peak braking/ propulsive forces, in addition to the active peak of the vertical ground reaciton force, increased linearly. This study also showed graded increases in knee/ ankle flexion and activation of the rectus femoris and soleus musculature. Naik et al., (5) examined 4, 6, 8, 10, and $12 \mathrm{~cm}$ heel heights during a stand-to-sit-returning task. Their findings showed imbalances between vastus lateralis and medialis muscles that bccame more prominent in elevated heels. Simonsen et al., (6), Esenyel et al., (7) and Kerrigan et al., (8) each found that the magnitude of knee extensor moment during the first half of the stance phase was substantially larger, which was attributed to increased knee flexion when wearing heeled footwear. In addition, several investigations have shown that the magnitude of the external knee adduction moment increased significantly in high-heeled footwear compared to flat shoes and also linearly with increases in heel height $(6,9,10)$. At the ankle joint, Barkema et al., (9) found that the peak ankle eversion moment during late stance phase was amplified linearly with increases in heel height. Both, Esenyel et al., (7) and 
70 Simonsen et al., (6) showed that the peak plantarflexion moment at the end of the stance

71 phase was significantly reduced in heeled footwear. Finally, at the hip joint Simonsen et al.,

72 (6) showed that the hip joint abductor moment was significantly larger when walking in high73 heels.

75 Though females regularly wear high-heeled shoes, it has been suggested that their continued utilization may lead to an increased incidence of chronic musculoskeletal pathologies. Importantly, previous analyses have shown that stance phase knee adduction moments were statistically larger when walking in high-heels $(6,9,10)$. Leading to the proposition that highheels may augment compressive knee joint loading, placing wearers at risk from tibiofemoral joint osteoarthritis. A musculoskeletal condition renowned for its increased prevalence in females (11). Importantly, biomechanical accommodations to high-heels have been shown to vary with experience in wearing heeled footwear (12). Csapo et al., (13) showed using axialplane magnetic resonance imaging that experienced wearers were associated with shortening of the triceps-surae muscle-tendon units, compared to in-experienced users. Leading to the notion that wearing experience may affect female's susceptibility to chronic musculoskeletal pathologies.

Previously highlighted analyses concerning the biomechanical effects of high-heeled footwear on compressive joint loads linked to the aetiology of osteoarthritis, have utilized joint moments as pseudo indices of global joint kinetics (14). Furthermore, there has yet to be a comparative examination of muscle tendon unit kinematics when wearing high-heels, owing to a lack of suitable measurement techniques capable of quantifying muscle mechanics. Importantly, Herzog et al., (15) showed that muscles are the primary contributors 
to lower extremity joint loading. Yet the complex role of muscles in controlling joint

biomechanics during human movement has received insufficient attention within the literature, possibly due to difficulties in calculating muscle kinetics and kinematics. However, advances in musculoskeletal modelling have led to the development of bespoke software which allows skeletal muscle force distributions and muscle tendon lengths to be simulated during movement using motion capture based data (16). To date, such approaches have not yet been utilized to explore biomechanical differences between high-heeled and traditional footwear.

The aims of the current investigation were therefore twofold. Firstly, to examine the effects of different heeled footwear heights on lower extremity joint loading and triceps-surae muscle tendon kinematics, using a simulation based approach. Secondly, to examine both experienced and in-experienced high-heel users, in order to determine whether wearing experience affects female's potential susceptibility to chronic musculoskeletal pathologies. The current investigation may provide further important information regarding the potential chronic effects of high-heeled footwear in experienced and in-experienced users.

\section{Methods}

\section{Participants}

Twenty four female participants (12 experienced high-heel wearers; age 30.54 \pm 5.55 years, height $1.65 \pm 0.08 \mathrm{~cm}$ and body mass $63.42 \pm 6.73 \mathrm{~kg}$ and 12 inexperienced high-heel wearers; age $29.24 \pm 4.78$ years, height $1.66 \pm 0.11 \mathrm{~cm}$ and body mass $65.27 \pm 5.98 \mathrm{~kg}$ ) volunteered to take part in this study. To be considered an experienced high-heel wearer, participants had to 
have worn heels with a minimum heel height of $5 \mathrm{~cm}$ at least five times a week for a

118 minimum of 2 years (11). All participants were free from pathology at the time of data

119 collection and provided written informed consent, in accordance with the principles outlined

120 in the Declaration of Helsinki. The procedure utilized for this investigation was approved; by

121 a university ethical committee (REF 637).

\section{Experimental footwear}

The footwear used during this study consisted of traditional footwear (New Balance 1260 v2; Figure 1a), high heels (10cm heel; Figure $2 \mathrm{~d})$, medium heels $(7 \mathrm{~cm}$ heel; Figure $2 \mathrm{c})$, and low heels $(4 \mathrm{~cm}$ heel; Figure $2 \mathrm{~b})$ in sizes $3-6$ in UK. The heeled-footwear were identical with the exception of the heel heights.

\section{Procedure}

Participants walked at a velocity of $1.5 \mathrm{~m} / \mathrm{s}( \pm 5 \%)$, striking an embedded piezoelectric force platform (Kistler, Kistler Instruments Ltd) with their right (dominant) foot. Walking velocity was monitored using infrared timing gates (Newtest, Oy Koulukatu). The stance phase was delineated as the duration over which $20 \mathrm{~N}$ or greater of vertical force was applied to the force platform. Participants completed a minimum of five successful trials in each footwear condition. The order that participants walked in each footwear condition was counterbalanced. Kinematics and ground reaction forces data were synchronously collected. 
139 Kinematic data was captured at $250 \mathrm{~Hz}$ via an eight camera motion analysis system (Qualisys

140 Medical $\mathrm{AB}$ ) and ground reaction forces captured at $1000 \mathrm{~Hz}$. Dynamic calibration of the

141 motion capture system was performed before each data collection session.

143 To define the anatomical frames of the thorax, pelvis, thighs, shanks and feet retroreflective 144 markers were placed at the C7, T12 and xiphoid process landmarks and also positioned 145 bilaterally onto the acromion process, iliac crest, anterior superior iliac spine (ASIS), 146 posterior super iliac spine (PSIS), medial and lateral malleoli, medial and lateral femoral 147 epicondyles, greater trochanter, calcaneus, first metatarsal and fifth metatarsal. Carbon-fiber 148 tracking clusters comprising of four non-linear retroreflective markers were positioned onto 149 the thigh and shank segments. In addition to these the foot segments were tracked via the calcaneus, first metatarsal and fifth metatarsal, the pelvic segment was tracked using the PSIS and ASIS markers and the thorax segment was tracked using the T12, C7 and xiphoid 152 markers.

154 Static calibration trials were obtained with the participant in the anatomical position in order 155 for the positions of the anatomical markers to be referenced in relation to the tracking clusters/markers. A static trial was conducted with the participant in the anatomical position

157 in order for the anatomical positions to be referenced in relation to the tracking markers, 158 following which those not required for dynamic data were removed. 
Dynamic trials were digitized using Qualisys Track Manager, in order to identify anatomical and tracking markers and then exported as C3D files to Visual 3D (C-Motion, Germantown, MD). All data were normalized to $100 \%$ of the stance phase. Ground reaction force and kinematic data were smoothed using cut-off frequencies of 12 and $6 \mathrm{~Hz}$ with a low-pass Butterworth 4th order zero lag filter (17). All net force parameters throughout were normalized by dividing by bodyweight (BW). Following this the external vertical rate of loading (BW/s) was quantified, as the peak increase in force between adjacent data points.

Data during the stance phase were exported from Visual 3D into OpenSim 3.3 software (Simtk.org). A validated musculoskeletal model with 12 segments, 19 degrees of freedom and 92 musculotendon actuators (18) was used to estimate lower extremity joint forces. The model was scaled for each participant to account for the anthropometrics of each. As muscle forces are the main determinant of joint compressive forces (15), muscle kinetics were quantified using a weighted static optimization in accordance with Steele et al., (19). Compressive ankle, medial/ lateral tibiofemoral and hip joint forces were calculated via the joint reaction analyses function using the muscle forces generated from the static optimization process as inputs. The joint reaction analysis function in OpenSim calculates the joint loads transferred between two contacting bodies, about the joint centre location identified during the static trial (19). In the current investigation, hip joint forces were representative of the sum of contact forces between the femur and acetabular cartilage, tibiofemoral forces between the medial/ lateral tibial and femoral cartilage and ankle joint forces between the tibia and talar cartilage. From the above processing, peak ankle force, peak medial tibiofemoral force, peak lateral tibiofemoral force and peak hip force were extracted for statistical analyses. In addition ankle, medial/ lateral tibiofemoral and hip 
instantaneous load rates (BW/s) were also extracted by obtaining the peak increase in force between adjacent data points.

Patellofemoral loading was quantified using a model adapted from van Eijden et al., (20). A key drawback of this model is that co-contraction of the knee flexor musculature is not accounted for (21). Taking this into account, summed hamstring and gastrocnemius forces derived from the static optimization procedure were multiplied by their estimated knee joint muscle moment arms as a function of knee flexion angle (22), and then added together to determine the knee flexor torque during the stance phase. In addition to this, the knee extensor torque was also calculated by dividing the summed quadriceps forces by this muscle groups' knee joint muscle moment arms as a function of knee flexion angle (van Eijden et al., (20). The knee flexor and extensor torques were then summed and subsequently divided by the quadriceps muscle moment arm to obtain quadriceps force adjusted for co-contraction of the knee flexor musculature. Patellofemoral force was quantified by multiplying the derived quadriceps force by a constant obtained by using the data of Eijden et al., (20). Finally, patellofemoral joint stress $(\mathrm{KPa} / \mathrm{BW})$ was quantified by dividing the patellofemoral force by the patellofemoral contact area. Patellofemoral contact areas were obtained by fitting a polynomial curve to the sex specific data of Besier et al., (23). From the above processing, peak patellofemoral force and peak patellofemoral stress were extracted for statistical analyses. In addition, patellofemoral instantaneous load rate (BW/s) was also extracted by obtaining the peak increase in force between adjacent data points.

Finally, Achilles tendon forces were estimated in accordance with the protocol of Almonroeder et al., (24), by summing the muscle forces of the medial gastrocnemius, lateral, 
gastrocnemius, and soleus muscles. From the above processing, peak Achilles tendon force and Achilles tendon instantaneous load rate (BW/s) were extracted for statistical analyses.

212 Heeled footwear may affect the number of footfalls required to complete a set distance. We 213 therefore firstly calculated integral of the hip, tibiofemoral, patellofemoral, ankle and 214 Achilles tendon forces during the stance phase, using a trapezoidal function. In addition to 215 this, we also estimated the total force per mile (BW·mile) by multiplying these parameters by the number of steps required to walk one mile. The number of steps required to complete one

217 mile was quantified using the step length (m), which was determined by taking the difference in the horizontal position of the foot centre of mass between the right and left legs at footstrike.

Muscle-tendon lengths were also determined using OpenSim in accordance with Sinclair, (25), via the positions of their proximal and distal muscle origins. The muscle tendon complexes which were evaluated as part of the current research were the Lateral gastrocnemius, Medial gastrocnemius and Soleus. The mean lengths of these muscle tendon units during the stance phase were extracted for statistical analysis.

Statistical analyses

Descriptive statistics of means and standard deviations were obtained for each outcome measure. Shapiro-Wilk tests were used to screen the data for normality. Differences in biomechanical parameters were examined using 4 (FOOTWEAR) x 2 (EXPERIENCE) mixed ANOVA's. In the event of a significant main effect pairwise comparisons were 
232 performed. Statistical significance was accepted at the $\mathrm{P} \leq 0.05$ level (26). Effect sizes for all 233 significant findings were calculated using partial $\mathrm{Eta}^{2}\left(\mathrm{p \eta}^{2}\right)$. In accordance with Sinclair et al., 234 (26) the minimum clinically important difference (MCID) was considered to be $2.3 *$ the 235 pooled standard error of measurement. All statistical actions were conducted using SPSS 236 v24.0 (SPSS Inc, Chicago, USA).

$238 \quad$ Results

239 Tables 1-2 and figures 2-3 present the joint load and muscle kinematics variables obtained as 240 a function of the different heel height conditions and experience in wearing high-heeled 241 footwear.

249 A main effect of FOOTWEAR was found for step length $\left(\mathrm{P}<0.05, \mathrm{p} \eta^{2}=0.51\right)$. Post-hoc pairwise comparisons showed that step length was significantly greater in the trainer, compared to the high, medium and low heels, and significantly larger in the medium and low 252 heels compared to the high heel condition (Table 1). 
254 A main effect of FOOTWEAR was found for the external vertical load rate $(\mathrm{P}<0.05$, $\left.255 \mathrm{p} \eta^{2}=0.50\right)$. Post-hoc pairwise comparisons showed that the load rate was significantly greater 256 in the high heels compared to the, medium, low and trainer conditions, and significantly 257 larger in the medium and low heels compared to the trainer (Table 1).

Hip joint loading

For the load experienced per mile, a main effect of FOOTWEAR $\left(\mathrm{P}<0.05, \mathrm{p} \eta^{2}=0.19\right)$ was observed. Post-hoc pairwise comparisons showed that the load experienced per mile was significantly larger in the high and low heels in comparison to the trainer (Table 1).

265 For medial tibiofemoral load rate, a main effect of FOOTWEAR $\left(\mathrm{P}<0.05, \mathrm{p} \eta^{2}=0.15\right)$ was observed. Post-hoc pairwise comparisons showed that the medial tibiofemoral load rate was significantly larger in the high heels in comparison to the low heels and trainer conditions (Table 1). For medial tibiofemoral load experienced per mile, a main effect of FOOTWEAR $\left(\mathrm{P}<0.05, \mathrm{p \eta}^{2}=0.23\right)$ was observed. Post-hoc pairwise comparisons showed that the load experienced per mile was significantly larger in the high, medium and low heel conditions in comparison to the trainer (Table 1).

273 For lateral tibiofemoral load experienced per mile, a main effect of FOOTWEAR $(\mathrm{P}<0.05$, $\left.274 \mathrm{p \eta}^{2}=0.24\right)$ was observed. Post-hoc pairwise comparisons showed that the load experienced 
per mile was significantly larger in the high heel in comparison to the medium, low and trainer conditions (Table 1).

\section{Patellofemoral joint loading}

Main effects of FOOTWEAR were observed for peak patellofemoral force $(\mathrm{P}<0.05$, $\left.\mathrm{p} \eta^{2}=0.70\right)$ and stress $\left(\mathrm{P}<0.05, \mathrm{p} \eta^{2}=0.68\right)$. Post-hoc pairwise comparisons showed that peak force and stress were significantly greater in the high, medium and low heels in comparison to the trainer, and significantly larger in the high heels compared to the medium and low heel conditions (Table 1; Figure 2de). In addition, a main effect of FOOTWEAR was observed for patellofemoral load rate $\left(\mathrm{P}<0.05, \mathrm{p \eta}^{2}=0.61\right)$. Post-hoc pairwise comparisons showed that load rate was significantly greater in the high, medium and low heels in comparison to the trainer, and significantly larger in the high heels compared to the medium and low heel conditions (Table 1). Finally, a significant main effect of FOOTWEAR was observed for patellofemoral force per mile $\left(\mathrm{P}<0.05, \mathrm{p \eta}^{2}=0.63\right)$. Post-hoc pairwise comparisons showed that each footwear differed significantly from one another, with the patellofemoral force per mile increasing linearly with increases in heel height (Table 1).

\section{Ankle joint loading}

Main effects of FOOTWEAR were observed for peak ankle force $\left(\mathrm{P}<0.05, \mathrm{p} \eta^{2}=0.60\right)$ and Achilles tendon force $\left(\mathrm{P}<0.05, \mathrm{p \eta}^{2}=0.82\right)$. Post-hoc pairwise comparisons showed that each footwear differed significantly from one another, with peak ankle force decreasing linearly with increases in heel height (Table 1; Figure 2fg). 
In addition, a significant main effect of FOOTWEAR was observed for ankle force per mile $\left(\mathrm{P}<0.05, \mathrm{p}^{2}=0.46\right)$. Post-hoc pairwise comparisons showed that ankle force per mile was significantly greater in the medium, low and trainer conditions compared to the high heels. Furthermore, it was also revealed that force per mile was significantly larger in the low heel and trainer compared to the medium condition (Table 1). Finally, a significant main of FOOTWEAR was observed for Achilles tendon force per mile $\left(\mathrm{P}<0.05, \mathrm{p} \eta^{2}=0.73\right)$. Post-hoc pairwise comparisons showed that each footwear differed significantly from one another, with Achilles tendon force per mile decreasing linearly with increases in heel height (Table $1)$.

\section{Muscle lengths}

There were FOOTWEAR main effects for the Soleus $\left(\mathrm{P}<0.05, \mathrm{p} \eta^{2}=0.80\right)$, Medial gastrocnemius $\left(\mathrm{P}<0.05, \mathrm{p}^{2}=0.85\right)$ and Lateral gastrocnemius $\left(\mathrm{P}<0.05, \mathrm{p} \eta^{2}=0.85\right)$. Post-hoc pairwise comparisons showed for each muscle, that each footwear differed significantly from one another, with the mean muscle lengths decreasing linearly with increases in heel height (Table 2; Figure 3a-c).

\section{Discussion}

The current study examines the effects of different high-heeled footwear heights on lower extremity joint loading and triceps-surae muscle tendon kinematics. To the authors knowledge this represents the first investigation to examine the biomechanics of high-heeled footwear using musculoskeletal simulation, and may provide more detailed information regarding the effects of high-heeled footwear in experienced and in-experienced users. 
322 The current investigation showed that compressive hip joint loading experienced per mile was significantly increased in the high and low heels in comparison the trainer. As no alterations in peak loading were observed in these conditions, it can be concluded that the increased loading was mediated as a function of the decreased step length. The initiation and progression of osteoarthritis is mediated through chronic compressive loading experienced at the joint itself (28). However, whilst the current investigation showed that there were statistical increases in compressive hip loading, the magnitude of the differences between footwear conditions did not exceed the MCID. This leads to the conclusion that heeled footwear may not influence wearers' susceptibility to chronic hip joint pathology, although further analysis should seek to confirm this notion.

In addition, the current investigation showed that compressive joint loading at both the medial and lateral aspects of the tibiofemoral joint were statistically influenced as a function of the different experimental footwear. At the medial tibiofemoral compartment, the load rate was larger in the high heels compared to the low heels and trainer conditions, and the load per mile was greater in each of the heeled footwear in compared to the trainer. However, only differences in force per mile between the high heels and trainer were beyond the MCID threshold. At the lateral compartment, the loads experienced per mile were greater in the high heel compared to the medium, low and trainer conditions, although the magnitude of these differences did not exceeded the MCID. Once again as no alterations in peak loading were observed, it can be concluded that increased loads per mile were mediated as a function of decreases in step length. However, the increased medial load rate in the heeled footwear is likely a consequence of the increased rate at which the external ground reaction force is 
experienced in these conditions. The medial tibiofemoral compartment is at much greater risk from degenerative joint osteoarthritis compared to the lateral aspect of the knee joint (29). As such it appears that irrespective of experience, the high heels increased the risk of medial knee osteoarthritis compared to the trainer, an observation in agreement with the propositions of Kerrigan et al., (10).

At the patellofemoral joint, compressive loading parameters were shown to generally increase linearly with increases in heel height, and predominantly exceeded the MCID between each footwear condition. Patellofemoral pain is one of the most common chronic musculoskeletal disorders of the lower extremities, and like osteoarthritis is more common in females compared to males (30). Patellofemoral pain may be the result of increased patellofemoral joint stress (31), and is thought longitudinally, to progress to patellofemoral joint osteoarthritis (32). The enhanced patellofemoral joint stress shown in the high-heeled footwear conditions was mediated by increases in the patellofemoral force, in particular as increases in knee flexion shown in the high-heeled footwear conditions (Supplemental data 1a) lead to increased patellofemoral contact areas (23). In turn it is proposed that the augmented patellofemoral force was caused by increases in knee extensor muscle forces, a key input parameter into the patellofemoral joint musculoskeletal model. Increased knee extensor muscle force requirements were mediated via a more posterior orientation of the ground reaction force vector in the high-heeled footwear (33). The current study therefore provides strong evidence that high-heeled footwear of increased height results in elevated patellofemoral joint stress, which could potentially lead to an increase in patellofemoral symptoms over time. 
It was also revealed that muscle tendon kinematics were significantly influenced as a function of different heel heights, and importantly that the magnitude of the differences exceeded the MCID in all cases. Specifically, the current study showed that each of the triceps-surae muscle tendon-unit lengths during the stance phase decreased linearly with increases in heel height. This investigation also showed that ankle and Achilles tendon loading also decreased linearly alongside increases in heel height, with the differences between footwear conditions surpassing the magnitude of the MCID in the majority of cases. This observation opposes previous suggestions $(5,6)$, who suggested that triceps-surae muscles forces are likely to increase when wearing high-heels. It is proposed that the decreased ankle and Achilles tendon loading can be explained concomitantly by the shorter triceps-surae muscle lengths, reduced Achilles tendon moment arm as a function of enhanced ankle plantar flexion, combined with a ground reaction force vector that passes closer to the ankle joint centre. These parameters serve to increase the forces generated by the triceps-surae muscles in the trainer condition (Supplemental data $1 \mathrm{bcd}$ ), which strongly govern the loads experienced compressively by ankle joint and are solely responsible for those experienced by the Achilles tendon. This finding does oppose the notion proposed by Csapo et al., (13) that increased Achilles tendon cross-sectional area revealed in experienced high-heel wearers is mediated via increases in the relative muscle forces acting on the tendon-aponeurosis complex. Future analyses should therefore seek to better understand examine the biomechanical mechanisms that promote Achilles tendon hypertrophy in regular high-heel wearers.

However, the linear reductions in muscle tendon lengths strongly support the findings of surae muscles in a chronically shortened position, and are attributable to the ankle being at an increasingly more plantarflexed angle. Acute shortening of the triceps-surae muscle tendon 
units during walking is energetically inefficient (13), as it causes unnecessary overlap of the actin-myosin units and forces the muscle fibers into a non-optimal operating range (34). Habitual shortening of the triceps-surae muscle tendon units through regular utilization of heeled-footwear mediates chronic muscle tendon unit adaptations, whereby the muscle itself is shortened by reducing the number of in-series sarcomeres in order to transfer the actinmyosin overlap back to optimal operating range (34).

Importantly, the current investigation also revealed that there were no statistical main effects for EXPERIENCE, nor were there any significant interactions between FOOTWEAR $\mathrm{x}$ EXPERIENCE. This observation concurs with those of Ebbeling et al., (34) and Simonsen et al., (6) yet opposes the observations of Barton et al., (36); de Oliveira Pezzan et al., (37); and Gefen et al. (38). Nonetheless, the current investigation has shown that heeled footwear is associated with increased compressive tibiofemoral and patellofemoral joint loading and also places each of the triceps-surae muscle tendon units in a shortened position during the stance phase. These parameters are linked to the aetiology of degenerative joint osteoarthritis (28), and chronic shortening of the triceps-surae muscle tendon units (13). As such the current investigation indicates that the potential chronic effects of heeled footwear of increasing heights, appear to be independent of the users experience in wearing high-heeled footwear.

In conclusion, although walking biomechanics in heeled-footwear has received previous research attention; there has yet to be a quantitative comparison of lower extremity joint loading/ muscle tendon kinematics, using a musculoskeletal simulation based approach. The present investigation adds to the current knowledge, by examining the effects of different high-heeled footwear heights on lower extremity joint loading and triceps-surae muscle 
tendon kinematics in experienced and in-experienced heel wearers. This investigation showed irrespective of experience, that compressive loading at the medial tibiofemoral and patellofemoral joints was enhanced beyond the MCID in high-heels of increasing height. Furthermore, irrespective of experience, the triceps-surae muscle tendon units were shown to be placed in a shortened position when wearing high-heels of increasing height, with the magnitude of the differences exceeding the MCID. It can therefore be concluded that heeledfootwear increase the mechanical factors linked to the aetiology of degenerative joint osteoarthritis and chronic shortening of the triceps-surae muscle tendon units. Therefore, the current investigation provides evidence that irrespective of experience, heeled-footwear of increasing height may negatively influence females' lower extremity musculoskeletal health.

\section{References}

1. Linder M, Saltzman CL. (1998). A history of medical scientists on high heels. Int J Health Serv 28: 201-225. doi:10.2190/GA2M-FLA2-17FB-V5PE

2. Hong WH, Lee YH, Chen HC, Pei YC, Wu, C. Y. (2005). Influence of heel height and shoe insert on comfort perception and biomechanical performance of young female adults during walking. Foot Ankle Int 26: 1042-1048. doi: $10.1177 / 107110070502601208$

3. Cronin NJ (2014). The effects of high heeled shoes on female gait: a review. J Electromyogr Kinesiol 24: 258-263. doi: 10.1016/j.jelekin.2014.01.004

4. Stefanyshyn DJ, Nigg BM, Fisher V, O'Flynn B, Liu W (2000). The influence of high heeled shoes on kinematics, kinetics, and muscle EMG of normal female gait. J App Biomech 16: 309-319. doi: https://doi.org/10.1123/jab.16.3.309 
5. Naik GR, Al-Ani A, Gobbo M, Nguyen HT. (2017). Does heel height cause imbalance during sit-to-stand task: surface EMG perspective. Frontiers Physiology, 8, 626-634.

6. Simonsen EB, Svendsen MB, Nørreslet A, Baldvinsson HK, Heilskov-Hansen T, Larsen PK, Henriksen M. (2012). Walking on high heels changes muscle activity and the dynamics of human walking significantly. J App Biomech 28: 20-28. doi: 10.1123/jab.28.1.20

7. Esenyel M, Walsh K, Walden JG, Gitter A (2003). Kinetics of high-heeled gait. J Am Podiatr Med Assoc 93: 27-32. doi: https://doi.org/10.7547/87507315-93-1-27

8. Kerrigan DC, Lelas JL, Karvosky ME (2001). Women's shoes and knee osteoarthritis. Lancet, 357: 1097-1098. doi: 10.1016/S0140-6736(00)04312-9

9. Barkema DD, Derrick TR, Martin PE (2012). Heel height affects lower extremity frontal plane joint moments during walking. Gait Posture 35: 483-488. doi: 10.1016/j.gaitpost.2011.11.013

10. Kerrigan DC, Todd MK, Riley PO (1998). Knee osteoarthritis and high-heeled shoes. Lancet 351: 1399-1401. doi: 10.1016/S0140-6736(97)11281-8

11. Hame SL, Alexander RA (2013). Knee osteoarthritis in women. Curr Rev Musculoskelet Med 6: 182-187.

12. Hapsari VD, Xiong S (2016). Effects of high heeled shoes wearing experience and heel height on human standing balance and functional mobility. Ergonomics 59: 249264. https://doi.org/10.1080/00140139.2015.1068956

13. Csapo R, Maganaris CN, Seynnes OR, Narici MV. (2010). On muscle, tendon and high heels. J Exp Biol 213: 2582-2588. doi: 10.1242/jeb.044271

14. Herzog W, Longino D, Clark A (2003a). The role of muscles in joint adaptation and degeneration. Langenbecks Arch Surg 388: 305-315. 
15. Herzog W, Clark A, Wu J (2003b). Resultant and local loading in models of joint disease. Arthritis Care Res 49: 239-247. doi: https://doi.org/10.1002/art.11004

16. Delp SL, Anderson FC, Arnold AS, Loan P, Habib A, John CT, Thelen DG (2007). OpenSim: open-source software to create and analyze dynamic simulations of movement. IEEE T Biomed Eng 54: 1940-1950. doi: 10.1109/TBME.2007.901024

17. Lerner ZF, Haight DJ, DeMers MS, Board WJ, Browning RC (2014). The effects of walking speed on tibiofemoral loading estimated via musculoskeletal modeling. J App Biomech 30: 197-205. doi: 10.1123/jab.2012-0206

18. Lerner ZF, DeMers MS, Delp SL, Browning RC (2015). How tibiofemoral alignment and contact locations affect predictions of medial and lateral tibiofemoral contact forces. J Biomech 48: 644-650. doi: 10.1016/j.jbiomech.2014.12.049

19. Steele KM, DeMers MS, Schwartz MH, Delp SL (2012). Compressive tibiofemoral force during crouch gait. Gait Posture 35: 556-560. doi: https://doi.org/10.1016/j.gaitpost.2011.11.023

20. Van Eijden TMGJ, Kouwenhoven E, Verburg J, Weijs WA (1986). A mathematical model of the patellofemoral joint. $\mathrm{J}$ Biomech 19: 219-229. doi: https://doi.org/10.1016/0021-9290(86)90154-5

21. Willson JD, Ratcliff OM, Meardon SA, Willy RW (2015). Influence of step length and landing pattern on patellofemoral joint kinetics during running. Scand J Med Sci 25: 736-743. doi: 10.1111/sms.12383

22. Spoor CW, Van Leeuwen JL (1992). Knee muscle moment arms from MRI and from tendon travel. J Biomech 25: 201-206.

23. Besier TF, Draper CE, Gold GE, Beaupré GS, Delp SL (2005). Patellofemoral joint contact area increases with knee flexion and weight-bearing. J Orthop Res 23: 345350. doi: 10.1016/j.orthres.2004.08.003 
24. Almonroeder T, Willson JD, Kernozek TW (2013). The effect of foot strike pattern on Achilles tendon load during running. Ann Biomed Eng 41: 1758-1766. doi: 10.1007/s10439-013-0819-1

25. Sinclair J. (2016). Side to side differences in hamstring muscle kinematics during maximal instep soccer kicking. Mov Sport Sci 91: 85-92. doi: 10.1051/sm/201502

26. Sinclair J, Taylor PJ, Hobbs S.J (2013). Alpha level adjustments for multiple dependent variable analyses and their applicability-a review. Int J Sports Sci Eng 7: 17-20.

27. Sinclair J, Janssen J, Richards JD, Butters B, Taylor PJ, Hobbs SJ. (2018). Effects of a 4-week intervention using semi-custom insoles on perceived pain and patellofemoral loading in targeted subgroups of recreational runners with patellofemoral pain. Phys Ther Sport 34: 21-27. doi: 10.1016/j.ptsp.2018.08.006.

28. Felson DT (2004). Risk factors for osteoarthritis: understanding joint vulnerability. Clin Orthop Relat Res 427: 16-21. doi: 10.1097/01.blo.0000144971.12731.a2

29. Vincent KR, Conrad BP, Fregly BJ, Vincent HK (2012). The pathophysiology of osteoarthritis: a mechanical perspective on the knee joint. PM\&R 4: 3-9. doi: 10.1016/j.pmrj.2012.01.020

30. Fulkerson JP, Arendt EA (2000). Anterior knee pain in females. Clin Orthop Relat Res 372: 69-73.

31. Heino JB, Powers CM (2002). Patellofemoral stress during walking in persons with and without patellofemoral pain. Med Sci Sports Exerc 34: 1582-1593. doi: 10.1249/01.MSS.0000035990.28354.c6

32. Thomas MJ, Wood L, Selfe J, Peat G (2010). Anterior knee pain in younger adults as a precursor to subsequent patellofemoral osteoarthritis: a systematic review. BMC Musc Dis 11: 201-205. doi: 10.1186/1471-2474-11-201 
33. Almonroeder TG, Benson LC, O’Connor KM (2015). Changes in patellofemoral joint stress during running with the application of a prefabricated foot orthotic. Int J Sports Phys Ther 10: 967-972.

34. Ebbeling CJ, Hamill J, Crussemeyer JA (1994). Lower extremity mechanics and energy cost of walking in high-heeled shoes. JOSPT 19: 190-196. doi: 10.2519/jospt.1994.19.4.190

35. Zöllner AM, Pok JM, McWalter EJ, Gold GE, Kuhl E (2015). On high heels and short muscles: a multiscale model for sarcomere loss in the gastrocnemius muscle. $\mathrm{J}$ Theor Biol 365: 301-310. doi: 10.1016/j.jtbi.2014.10.036

36. Barton CJ, Coyle JA, Tinley P (2009). The effect of heel lifts on trunk muscle activation during gait: a study of young healthy females. J Electromyogr Kinesiol 19: 598-606. doi: 10.1016/j.jelekin.2008.03.001

37. de Oliveira Pezzan PA, João SMA, Ribeiro AP, Manfio EF (2011). Postural assessment of lumbar lordosis and pelvic alignment angles in adolescent users and nonusers of high-heeled shoes. J Manipulative Physiol Ther 34: 614-621. doi: 10.1016/j.jmpt.2011.09.006

38. Gefen A, Megido-Ravid M, Itzchak Y, Arcan M (2002). Analysis of muscular fatigue and foot stability during high-heeled gait. Gait Posture 15: 56-63. doi: https://doi.org/10.1016/S0966-6362(01)00180-1

\section{List of figures}

Figure 1: Experimental footwear $(\mathrm{a} .=$ trainer, $\mathrm{b} .=$ low heel, $\mathrm{c} .=$ medium heel and d. $=$ high heel). 
539 Figure 2: Joint loading lengths as a function of different heel heights and experience. $($ a. $=$ 540 hip, b. = medial tibiofemoral, c. = lateral tibiofemoral, d. = patellofemoral force, e. $=$ 541 patellofemoral stress, f. = Achilles tendon, g. = ankle). (black = high heel, light grey =

542 medium heel, black dot $=$ low heel, dark grey $=$ trainer, black dash $=$ high heel experienced,

543 black outline $=$ medium heel experienced, grey dot $=$ low heel experienced and dark grey 544 outline $=$ trainer experienced).

545 Figure 3: Muscle tendon lengths as a function of different heel heights and experience $(\mathrm{a} .=$ 546 Soleus, b. = Lateral gastrocnemius, c. $=$ Medial gastrocnemius). (black $=$ high heel, light grey $547=$ medium heel, black dot $=$ low heel, dark grey $=$ trainer, black dash $=$ high heel experienced, 548 black outline $=$ medium heel experienced, grey dot $=$ low heel experienced and dark grey 549 outline $=$ trainer experienced).

\section{$\underline{\text { Supplemental data }}$}

552 Appendix Figure 1: (a. = knee flexion angle during the stance phase, $\mathrm{b} .=$ Soleus muscle 553 force, c. = Lateral gastrocnemius muscle force, $\mathrm{d}$. = Medial gastrocnemius muscle force) . 554 (black $=$ high heel, light grey $=$ medium heel, black dot $=$ low heel, dark grey $=$ trainer, black 555 dash $=$ high heel experienced, black outline $=$ medium heel experienced, grey dot $=$ low heel experienced and dark grey outline $=$ trainer experienced) 